\title{
Splenic infarction in a man with active drug use
}

\section{Abstract}

Splenic infarction is a common finding in patients of infective endocarditis due to septic emboli of the spleen. However, the occurrence of acute abdominal pain as an initial manifestation of endocarditis is unusual. Herein, we report a case of splenic infarct and infective endocarditis caused by Streptococcus constellatus cured by antibiotic treatment and valve replacement without the aid of splenic intervention.

\author{
Volume 6 Issue 6 - 2017
}

\section{Fadi Al Akhrass, Lina Abdallah, Krishna Ammisetty, Muhannad Antoun}

Department of Infectious Diseases and Infection Control, Pikeville Medical Center, USA

\author{
Correspondence: Fadi Al Akhrass, Medical director of \\ Infectious Diseases and Infection Control, Pikeville Medica \\ Center, USA, Tel 606-794-6416, \\ Email fadi.akhrass@pikevillehospital.org
}

Received: May 23, 2017 | Published: May 31, 2017

\section{Introduction}

Splenic infarction is the result of ischemic event to the spleen. The infarct may be segmental or global. The arterial supply to the spleen consists of the splenic artery and the short gastric arteries. ${ }^{1}$ There are numerous etiologies of splenic infarct. The vast majority are either infiltrative hematologic diseases that cause congestion of the splenic circulation by abnormal cells, or thromboembolic conditions that produce obstruction of larger vessels. Splenic infarction of endovascular origin occurs when the splenic artery or one or more of its sub-branches become occluded with an infected or bland embolus or clot. ${ }^{2}$ Streptococcus anginosus group is widely known for its ability to cause invasive pyogenic infections. ${ }^{3}$ Isolation of Streptococcus anginosus group from the blood should prompt careful evaluation for the presence of one or more sites of focal suppurative infection, including infective endocarditis (IE).

\section{Case report}

A 31-year-old Caucasian man presented to the hospital with 4day-history of left upper quadrant abdominal pain and left sided pleurisy. He had recently failed therapy for chronic HCV monoinfection, genotype 1a, with fibrosis score of F0. He admits to using intravenous recreational drugs. Vital signs were normal except for heart rate of $130 / \mathrm{min}$. The abdomen was tender in the left upper abdominal quadrant with no of peritoneal irritation. Initial blood investigations revealed leukocytosis of $21.3 \times 103 / \mu 1$ with normal renal function and liver enzymes. Computed tomography of the abdomen with IV contrast showed a large splenic infarct (Figure 1). He had no recent abdominal trauma or abdominal surgery. Streptococcus constellatus was cultivated from two sets of blood cultures on day three. Comprehensive workup excluded mononucleosis, pancreatic disorders, HIV, autoimmune/collagen vascular disease, sickle hemoglobinopathies, malignant hematologic disorders (normal peripheral blood smear, SPEP and flow cytometry), hepercoagulable state (normal protein $\mathrm{C}$ or protein $\mathrm{S}$; normal lupus anticoagulant, factor $\mathrm{V}$, prothrombin). Patient had no signs of ARDS, sepsis, septic shock or toxic shock syndrome. Patient no recent cardiac catheterization (angiographic embolization), sclerotherapy of esophageal varices, abdominal surgery (pancreatectomy, or liver transplant), vasopressin infusion, or embolization for splenic hemorrhage. Echocardiogram showed the presence of $2.5 \times 1.5 \mathrm{~cm}^{2}$ vegetation of the aortic valve with perforation, severe regurgitation and aortic root abscess (Figure 2). Magnetic resonance imaging (MRI) of the brain showed numerous brain abscesses, without mass effect or midline shift. This case was complex and Duke's criteria were used to make the diagnosis of IE. The patient received aortic valve replacement and his post-operative course was uneventful. Administration of ertapenem was continued for 8 weeks. The patient had full neurological recovery and repeat MRI of the brain showed complete resolution of the abscesses. Follow-up echocardiogram showed no thrombosis or vegetation of the bioprosthetic valve.

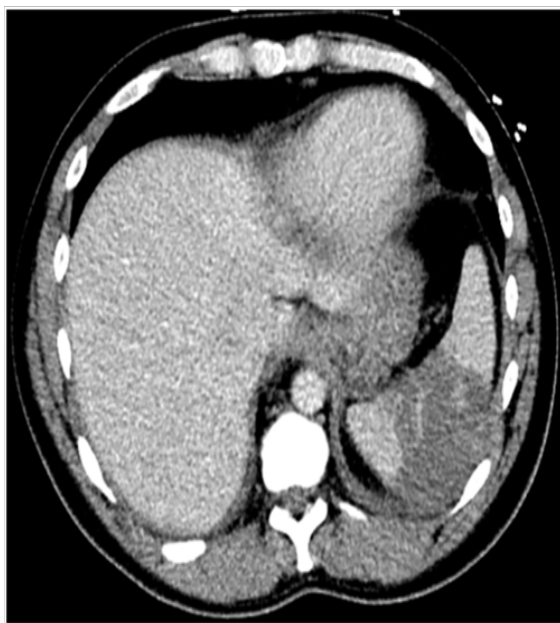

Figure IA) Computed tomography of the abdomen.

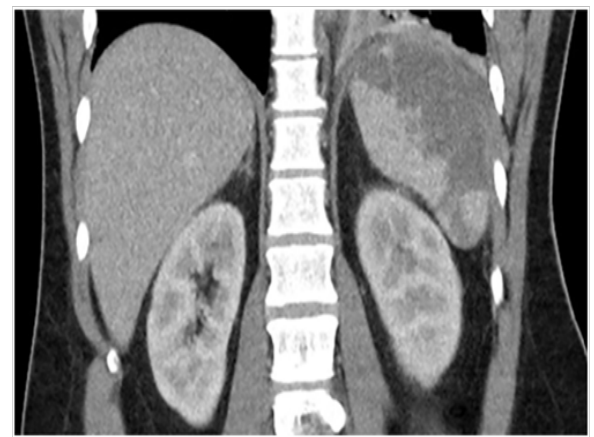

Figure IB 


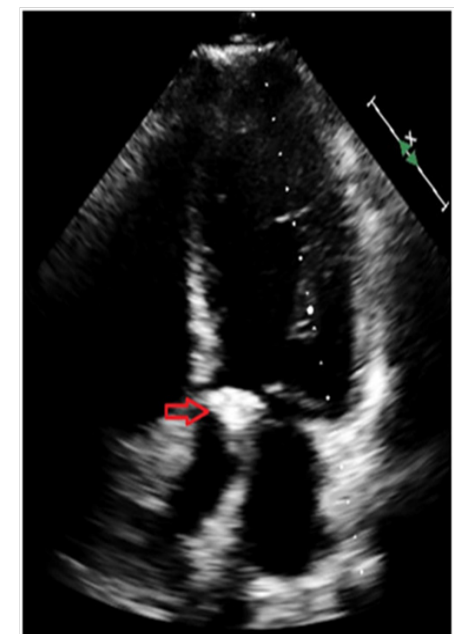

Figure 2 Echocardiogram showed the presence of $2.5 \times 1.5 \mathrm{~cm}^{2}$ vegetation of the aortic valve.

\section{Discussion}

The clinical spectrum of splenic infarction ranges from asymptomatic incidental infarction to hemorrhagic shock. Approximately one third of splenic infarcts are clinically occult. In a 10-year retrospective study, the most common symptom was either abdominal or left flank pain ( $80 \%$ of episodes), while the most common sign was left-upper-quadrant tenderness (35\% of episodes). ${ }^{4}$ Additional symptoms include fever and chills, nausea and vomiting, pleuritic chest pain, and left shoulder pain (Kehr sign). In a study of 26 patients with splenic infarction seen at a single medical center, leukocytosis was not a constant finding and was elevated in only $56 \%$ of the cases. ${ }^{5}$ A report on 108 patients with left-side IE undergoing valvular surgery revealed a $19 \%$ incidence of splenic infarction; in almost half of the patients with infarction, the diagnosis was made incidentally on computed tomography (CT). ${ }^{6}$ Delayed-phase contrastenhanced CT is the current diagnostic modality of choice of splenic infarct. Magnetic resonance imaging (MRI), preferably performed with IV gadolinium contrast, is another useful modality that clearly identifies infarcted splenic parenchyma. ${ }^{7}$ Ultrasonographic imaging is a less useful modality to diagnose an acute splenic infarct cases $(18 \%$ of patients). ${ }^{4}$

Infective endocarditis is associated with a broad array of complications (cardiac, neurologic, renal, and musculoskeletal) that can occur at any time during the illness. More than one complication can occur simultaneously. Embolization with clinical sequelae has been described in $13-44 \%$ of patients with IE; in most cases, embolization occurs prior to clinical presentation but can occur after initiation of antimicrobial therapy. ${ }^{8}$ Emboli can occlude or damage virtually any vessel in the systemic or pulmonary arterial circulation. ${ }^{9}$ As a result, embolization can cause stroke, Paralysis, blindness, ischemia of the extremities, splenic or renal infarction, pulmonary embolism, and acute myocardial infarction. Endocarditis should be considered as a possible etiology in all patients who present with signs or symptoms of systemic arterial embolization. Risk factors for embolization include presence of left-sided vegetation and more frequently with mitral vegetations than aortic vegetations ( $25 \%$ versus $10 \%)$, large vegetation size $(>1 \mathrm{~cm})$, microbiology (Staphylococcus aureus, Streptococcus bovis and fungal pathogens), presence of antiphospholipid antibodies, age, diabetes, atrial fibrillation, and embolization prior to initiation of antibiotics. ${ }^{9}$ Antimicrobial therapy has major impact on the incidence of stroke. In one study including 1437 patients with left-sided IE receiving appropriate therapy, the incidence of stroke fell from 4.8 to 1.7 per 1000 patient days between the first and second week of therapy. ${ }^{10}$ Development of metastatic abscess occurs as a sequela of septic embolization. Patients with splenic abscess may only present with persistent fever and/or recurrent bacteremia during or after completion of antimicrobial therapy. ${ }^{11}$ Splenic abscess frequently requires splenectomy for cure because of significantly improved outcome (mortality rate of $18 \%$ versus $100 \%$ in patients who received splenectomy compared to patients who did not undergo splenectomy). ${ }^{12,13}$ Mycotic aneurysm (infected aneurysm) can develop in the cerebral or systemic circulation in the setting of IE, usually at points of vessel bifurcation. ${ }^{13}$ The frequency of detected cerebrovascular complications in IE is increasing. In one study including 60 patients with IE, clinical signs and symptoms of cerebral embolism were observed in $35 \%$ of cases; clinically silent emboli were detected on MRI in an additional $30 \%$ of patients. ${ }^{14}$ Patient outcomes after a neurologic complication of IE are variable. It seems that staphylococcus aureus IE and neurologic manifestations are associated with high mortality of $74 \% .{ }^{15}$ Patient mortality in another series including 68 patients with stroke and IE was $50 \%$ at one year. ${ }^{16}$ In one study including 214 patients who underwent cardiac surgery for IE, $70 \%$ of patients with a preoperative stroke had full neurologic recovery, outcomes were worse in patients with stroke complicated by meningitis, abscess, or intracerebral hemorrhage. ${ }^{17}$

The Streptococcus anginosus group (previously known as the S. milleri group) is a subgroup of viridans streptococci that consists of three distinct streptococcal species: S. anginosus, S. intermedius, and S. constellatus. ${ }^{3}$ The group resides as part of the normal flora in the oral cavity and the gastrointestinal tract in humans. The distinctive characteristic of these organisms is the ability to cause abscesses and systemic infections which can be polymicrobial. ${ }^{18}$ Unlike less virulent members of the viridans streptococci, members of the S. anginosus group should be considered true pathogens when isolated from humans. ${ }^{19}$ Infections range from minor oral infections such as pharyngitis or dental abscess to life-threatening invasive infection with bacteremia and distant focal suppurative infection involving the lung, brain, liver, kidney, or soft tissues. Isolation of Streptococcus anginosus group from the blood should prompt careful evaluation for the presence of one or more sites of focal suppurative infection, including infective endocarditis. Among streptococcal isolates from patients with endocarditis, the S. anginosus group accounts for 3-15\% of cases..$^{20}$ Endocarditis caused by members of the $\mathrm{S}$. anginosus group is relatively uncommon, even in the presence of high-grade bacteremia. ${ }^{21}$ When endocarditis does occur, it is more common in patients with damaged or prosthetic heart valves than patients with normal native valves. This was not the case in our patient presentation and it seems that Streptococcus anginosus seems to be an emerging pathogen of native valve infective endocarditis in IV drug users. In a review of 29 patients with infective endocarditis due to $\mathrm{S}$. anginosus group isolates, cardiac surgery was required in $62 \%$ of the cases; predictors included acute valve dysfunction and intracardiac abscess..$^{22}$ In addition, distant focal suppurative complications requiring surgical drainage were observed in $25 \%$ of cases. ${ }^{22}$ In the absence of endocarditis or distant focal suppurative complications, bacteremia has a good prognosis. Treatment of infections caused by members of the S. anginosus group should include antimicrobial therapy as well as surgery for debridement/drainage. ${ }^{20}$ Ceftriaxone is the preferred agent due to excellent activity, infrequent dosing, and excellent tissue penetration. Metronidazole or clindamycin should be added for treatment of concomitant anaerobic infection. Alternatively, monotherapy with 
a beta-lactam-beta-lactamase inhibitor or a carbapenem are also reasonable choices for antimicrobial therapy. If allergy or resistance precludes use of beta-lactam agents, vancomycin is an appropriate alternative agent in patients with beta-lactam allergy (MICs 0.0125 to $1.0 \mathrm{mcg} / \mathrm{mL}$ ). The duration of therapy depends on the nature of the infection; in general, antimicrobial therapy should be administered until clinical signs of infection have resolved. Fluoroquinolone MICs among S. anginosus group members are high but in the susceptible range $(0.5$ to $1.0 \mathrm{mcg} / \mathrm{mL})$; resistance tends to develop easily, and therefore fluoroquinolones are not appropriate for treatment of patients with infections due to these organisms. ${ }^{23}$ Macrolide resistance appears to be emerging among the $\mathrm{S}$. anginosus group. ${ }^{24}$ Most strains of the $\mathrm{S}$. anginosus group are resistant to aminoglycosides. ${ }^{25}$ Sulfonamides have no activity against $\mathrm{S}$. anginosus group isolates.

Spleen surgical intervention is indicated only in the presence of complications (such as sepsis, abscess, or hemorrhage). The role of antiplatelet agents has not been formally addressed. Treatment of infections caused by members of the $\mathrm{S}$. anginosus group should include antimicrobial therapy as well as surgery for debridement/ drainage.

\section{Acknowledgements}

None.

\section{Conflict of interest}

The author declares no conflict of interest.

\section{References}

1. O'Keefe JH Jr, Holmes DR Jr, Schaff HV, et al. Thromboembolic splenic infarction. Mayo Clin Proc. 1986;61(12):967-972.

2. Antopolsky M, Hiller N, Salameh S, et al. Splenic infarction: 10years of experience. Am J Emerg Med. 2009;27(3):262-265.

3. 3-Claridge JE, Attorri S, Musher DM, et al. Streptococcus intermedius, Streptococcus constellatus, and Streptococcus anginosus ("Streptococcus milleri group") are of different clinical importance and are not equally associated with abscess. Clin Infect Dis. 2001;32(10):1511-1515.

4. Antopolsky M, Hiller N, Salameh S, et al. Splenic infarction: 10years of experience. Am J Emerg Med. 2001;27(3):262-265.

5. Lawrence YR, Pokroy R, Berlowitz D, et al. Splenic infarction: an update on William Osler's observations. Isr Med Assoc J. 2010;12(6):362365 .

6. Ting W, Silverman NA, Arzouman DA, et al. Splenic septic emboli in endocarditis. Circulation. 1990;82(5 Suppl):IV105-109.

7. Urban BA, Fishman EK. Helical CT of the spleen. AJR Am J Roentgenol. 1998;170(4):997-1003.

8. Hoen B, Duval X. Infective endocarditis. N Engl J Med. 2013;369:785.

9. Baddour LM, Wilson WR, Bayer AS, et al. Infective endocarditis in adults: diagnosis, antimicrobial therapy, and management of complications: a scientific statement for healthcare professionals from the american heart association. Circulation. 2015;132(15):1435-1486.
10. Dickerman SA, Abrutyn E, Barsic B, et al. The relationship between the initiation of antimicrobial therapy and the incidence of stroke in infective endocarditis:an analysis from the ICE Prospective Cohort Study (ICE-PCS). Am Heart J. 2007;154(6):1086-1094.

11. Weinstein L. Life-threatening complications of infective endocarditis and their management. Arch Intern Med. 1986;146(5):953-957.

12. Robinson SL, Saxe JM, Lucas CE, et al. Splenic abscess associated with endocarditis. Surgery. 1992;112(4):781-786.

13. Bisdas T, Teebken OE. Mycotic or infected aneurysm? Time to change the term. Eur J VascEndovascSurg. 2011;41(4):570-571.

14. Snygg-Martin U, Gustafsson L, Rosengren L, et al. Cerebrovascular complications in patients with left-sided infective endocarditis are common:a prospective study using magnetic resonance imaging and neurochemical brain damage markers. Clin Infect Dis. 2008;47(1):23-30.

15. Røder BL, Wandall DA, Espersen F, et al. Neurologic manifestations in Staphylococcus aureus endocarditis:a review of 260 bacteremic cases in nondrug addicts. Am J Med. 1997;102(4):379-386.

16. Anderson DJ, Goldstein LB, Wilkinson WE, et al. Stroke location, characterization, severity, and outcome in mitral vs aortic valve endocarditis. Neurology. 2003;61(10):1341-1346.

17. Ruttmann E, Willeit J, Ulmer H, et al. Neurological outcome of septic cardioembolic stroke after infective endocarditis. Stroke. 2006;37(8):2094-2099.

18. Shinzato T, Saito A. A mechanism of pathogenicity of "Streptococcus milleri group" in pulmonary infection: synergy with an anaerobe. $\mathrm{J} \mathrm{Med}$ Microbiol. 1994;40(2):118-123.

19. Doern CD, Burnham CA. It's not easy being green: the viridans group streptococci, with a focus on pediatric clinical manifestations. J ClinMicrobiol. 2010;48(11):3829-3825.

20. Kowlessar PI, O'Connell NH, Mitchell RD, et al. Management of patients with Streptococcus milleri brain abscesses. I Infect. 2006;52(6):443-450.

21. Bert F, Bariou-Lancelin M, Lambert-Zechovsky N. Clinical significance of bacteremia involving the "Streptococcus milleri" group: 51 cases and review. Clin Infect Dis. 1998;27(2):385-387.

22. Lefort A, Lortholary O, Casassus P. Comparison between adult endocarditis due to beta-hemolytic streptococci (serogroups A, B, C, and G) and Streptococcus milleri: a multicenter study in France. Arch Intern Med. 2002;162:2450.

23. Yamamoto N, Fujita J, Shinzato T. In vitro activity of sitafloxacin compared with several fluoroquinolones against Streptococcus anginosus and Streptococcus constellatus. Int J Antimicrob Agents. 2006;27(2):171-173.

24. Asmah N, Eberspächer B, Regnath T, et al. Prevalence of erythromycin and clindamycin resistance among clinical isolates of the Streptococcus anginosus group in Germany. J Med Microbiol. 2009;58(2):222-227.

25. Stelzmueller I, Biebl M, Berger N, et al. Relevance of group Milleri streptococci in thoracic surgery: a clinical update. Am Surg. 2007;73(5):492-497. 